\title{
IV. Die Bedeutung von Dietrich Bonhoeffers ethischen Überlegungen für die Medizinethik
}

Die ethische Diskussion um Zwangssterilisation und "Euthanasie“ war 1945 nicht zu Ende. Noch in den fünfziger und sechziger Jahren haben verschiedene Theologen und Psychiater Zwangssterilisationen gefordert ${ }^{1}$. Inzwischen hat sich jedoch die Einsicht verbreitet, daß dies mit dem Recht auf körperliche Unversehrtheit nach Art. 2 II GG unvereinbar wäre und außerdem eine Bekämpfung rezessiv erblicher Krankheiten durch Zwangssterilisation unmöglich ist. Heute noch diskutiert wird vor allem die Sterilisation nicht-einwilligungsfäbiger Frawen. Sie war unter gewissen Kautelen in der DDR zulässig ${ }^{2}$ und ist es in der vereinigten Bundesrepublik Deutschland nach Genehmigung durch einen Ausschuß, wenn andere Mittel der Empfängnisverhütung ungeeignet erscheinen. Kriterium ist nicht eine etwaige Erbkrankheit, sondern ein behauptetes Eigeninteresse geistig behinderter Frauen, keine Kinder zu bekommen, weshalb diese Sterilisation verfassungsrechtlich auch als Wahrnehmung eines Grundrechtes durch Dritte (Erziehungsberechtigte, Pfleger o.ä.) gerechtfertigt wird ${ }^{3}$. Einer solchen Vorgehensweise ist zugute zu halten, daß sie in ganz anderer Weise als in den dreißiger und vierziger Jahren vorstellbar auch geistig Behinderten Sexualkontakte ermöglichen möchte. Auch erinnert sie weniger an das nationalsozialistische Sterilisationsgesetz als vielmehr an die in den dreißiger Jahren in Schweden oder im Kanton Vaud/Schweiz üblichen Gesetze 4 . Gleichwohl liegt es auch auf der Hand, daß derartige Sterilisationen mit einem „Recht auf Fortpflanzung“, wie es Dietrich Bonhoeffer formulierte, nicht vereinbar sind.

Eine ähnliches Bild ergibt sich hinsichtlich der „Euthanasie", obwohl hier der Grad an Zustimmung immer schon geringer war als bezüglich der Zwangssterilisation. Noch in den fünfziger Jahren wurde in einem der maßgeblichen Grundgesetzkommentare die Menschenwürde im Rahmen einer Werttheorie der Grundrechte so verstanden, daß sie einen „Wertträger“ mit einer „Fähigkeit zu geistig-seelischem Empfinden" voraussetzte, woraus dann folgen sollte, daß "monstra“, geistig Schwerstbehinderten diese Fähigkeit abgehe, ihnen keine Menschenwürde zukomme und ihrer Tötung keine verfassungsrechtlichen Be-

${ }^{1}$ Vgl. Trillhaas, siehe S.15f. Anm.46; Nachtsheim, siehe S.114 Anm.302; Kolle, siehe S.95 Anm. 211; Schulte, siehe S. 104 Anm. 259.

${ }^{2}$ Vgl. Sozialistisches Gesundheitsrecht, S. 295.

${ }^{3}$ Zur kontroversen juristischen Debatte vgl. Wunder, Sterilisation; Eser/Koch, Rechtsprobleme; Hirsch/Hiersche, Sterilisation; Finger, Zulässigkeit.

4 Siehe S. 22 Anm. 8, und S. 48 Anm. 203. 
denken entgegenstünden ${ }^{5}$. Heute wird Vergleichbares in keinem Kommentar mehr vertreten. Diskutiert wird allenfalls ein Recht auf Selbsttötung und die Beihilfe dazu ${ }^{6}$. Derartige Überlegungen reagieren auf die durch die moderne Medizin enorm gesteigerte Möglichkeit, den Tod sterbender Menschen auf der Intensivstation hinauszuzögern, eine oft mehr als inhumane Behandlung. Doch erscheint dies kein Grund, die Unterscheidung zwischen einer sittlich verbotenen Tötung Kranker und einem unter gewissen Umständen zulässigen, vielleicht sogar sittlich gebotenen Sterbenlassen aufzugeben. Ähnlich argumentieren beispielsweise die beiden großen deutschen Kirchen', die der durch die moderne Intensivmedizin gewandelten Gesamtsituation vor allem durch Intensivierung der Schmerzbekämpfung (Palliativmedizin) und Humanisierung des Sterbens (Hospize u. a.) entgegenwirken wollen.

Im folgenden soll davon ausgegangen werden, daß Zwangssterilisation und "Euthanasie“ im nationalsozialistischen Sinn in seriösen Ethiken der Gegenwart nicht mehr gerechtfertigt werden. Insofern braucht nicht mehr die materialethische Konkretion, wohl aber deren Begründung diskutiert zu werden. Dies soll in der Form geschehen, daß die Bedeutung von Dietrich Bonhoeffers ethischen Überlegungen für die Medizinethik erörtert wird. Hierzu greifen wir nun auf die in der Ethik Dietrich Bonhoeffers formulierten Grundbegriffe zurück. In der Entfaltung seiner Verantwortungsethik sowie seiner These der Rechte natürlichen Lebens nahmen wir zugleich Grundgedanken Karl Bonhoeffers mit auf.

\section{Konsequenzen aus der Verantwortungsethik}

Dietrich Bonhoeffers "Struktur verantwortlichen Lebens" beginnt mit der Dimension der "Stellvertretung". Diese steht nicht zufällig am Anfang, denn dem stellvertretenden Handeln Gottes als Grundlage der zwischenmenschlichen Stellvertretung kommt in diesem ethischen Konzept eine grundlegende Bedeutung zu. Uns stellt sich nun die Aufgabe, diesen Gedanken für die Beziehung von Arzt/Ärztin und Patient/Patientin fruchtbar zu machen.

Die zwischenmenschliche Stellvertretung zielt nach Bonhoeffer auf eine Beziehung zweier Menschen, welche a) zwar ungleich angelegt, aber doch b) auf intensive Kommunikation beider ausgerichtet ist.

a) Als Beispiele nennt Bonhoeffer den Vater, den Staatsmann, den Lehrer, welcher für andere - Jüngere, Untergebene - Verantwortung übernimmt. Wir konnten das Beispiel des Arztes dem ohne weiteres hinzufügen. Dies alles sind Beispiele, die eine Vorrangstellung des Verantwortlichen bezüglich Verantwortungsbereitschaft, Einfluß und persönlicher Autorität voraussetzen, „Rollen also, die

\footnotetext{
${ }^{5}$ Vgl. Klein, in: Mangold/Klein, Grundgesetz I, zu Art. 1 GG, S. 148.

6 Vgl. Podlech, in: Azzola, Kommentar, zu Art. 1 I GG Rz. 55, S. 218.

7 Vgl. die Denkschrift Gott ist ein Freund des Lebens, S. 105-110.
} 
gerade durch Momente der Asymmetrie und der Einseitigkeit bestimmt sind " . Bonhoeffer kann sich eine Frau in dieser Position offensichtlich nicht vorstellen - eine Tatsache, die, selbst wenn man die Vorkriegssituation berücksichtigt, dem Theologen ein durchweg paternalistisches Denken bescheinigt ${ }^{9}$. Auch anderswo sieht man Bonhoeffer in der Gefahr, irdische Herrschaftsverhältnisse zu legitimieren oder sich jedenfalls mit ihnen abzufinden: „Gehorsams- und Abhängigkeitsverhältnisse wird es immer geben. Es kommt nur darauf an, daß sie nicht, wie es heute schon weithin ist, die Verantwortlichkeit aufheben. Es ist schwerer für den sozial Abhängigen, sich verantwortlich zu wissen, als für den sozial Freien. Aber keinesfalls schließt ein Abhängigkeitsverhältnis an sich schon die freie Verantwortung aus. Herr und Diener können und sollen in der Wahrung des Gehorsamsverhältnisses in freier Verantwortung für einander stehen." (S.268) Wir müssen es dazu bei einer kritischen Feststellung belassen und weitergehend fragen, inwiefern das Stellvertretungsmodell generell ungleichgewichtige oder gar paternalistische Züge trägt.

b) Eine korrigierende Vorstellung ist diesem Modell insofern inhärent, als die Vollmacht menschlichen Handelns für den Verantwortlichen nur so weit gilt, daß er „das Ich mehrerer Menschen in sich vereinigt" (S.257), es also versteht, deren Wollen und Wünschen, ja ihr Sein in die eigene Urteilsfindung mit aufzunehmen. Damit ist ein „In-Obhut-Nehmen“, welche dem anderen die Verantwortung nimmt, ausgeschlossen. Bonhoeffer beschreibt Verantwortung als ein Verhältnis der Gegenseitigkeit, der Reziprozität, auch wenn darin ein Moment der Einseitigkeit steckt, welches nicht vollständig an die Erfahrungen der Gegenseitigkeit gebunden ist. Diese beiden Pole lassen sich schon in der Christologie festmachen: Das stellvertretende Handeln Christi setzt nicht die Gegenseitigkeit als eine vom Menschen $\mathrm{zu}$ erbringende Vorgabe voraus, zielt aber doch auf Reziprozität im Sinne einer menschlichen Antwort. Ebenso wird auch im stellvertretenden menschlichen Handeln voraussetzungslos „für“ einen anderen Menschen gehandelt, in der Hoffnung, damit auch dessen Reaktionen zu verändern. „Wie solches einseitige und zuvorkommende Handeln möglich wird, ist das große Thema der Bergpredigt ${ }^{\text {"10 }}$, die Bonhoeffer (im Gegensatz zu Weber) ${ }^{11}$ nicht auf die Seite der Gesinnungsethik, sondern der Verantwortungsethik stellt, ohne dabei Gewaltlosigkeit als ein um jeden Preis durchzuhaltendes Prinzip anzusehen. In der gesellschaftspolitischen Dimension beinhaltet diese Stellvertretung zugleich einzutreten für die, die keine Stimme haben, und stellvertretend zu handeln für die, denen selbständiges Handeln verwehrt ist, die „geringsten Brüder“ (und Schwestern).

Für die Medizinethik ist dieses polare Modell der Stellvertretung ein interessanter Ansatz, weil das Konzept die Möglichkeit bietet, die faktische Asymme-

\footnotetext{
${ }^{8}$ Vgl. Huber, Sozialethik, S. 145.

9 Zum Frauenbild Bonhoeffers vgl. Siegele-Wenschkewitz, „Ehre“.

${ }_{10}$ Huber, Sozialethik, S. 145.

11 Vgl. Weber, Politik, S.551.
} 
trie der Arzt-Patient-Beziehung einerseits als gegeben und der Sache nach als unvermeidlich, auch als sinnvoll anzuerkennen, andererseits diese Ungleichheit konsequent, wiederholt und dringlich auf die in ihr mitgesetzte Wechselseitigkeit zu beziehen. Hier liegt der Überschuß, den Bonhoeffers relationale Verantwortungsethik gegenüber allen einseitig die Freiheit des Einzelnen betonenden Bestimmungen der Ethik enthält (wie etwa die Definition der Verantwortung als "Antwort der eigenen Lebensführung") ${ }^{12}$. Das Konzept der Stellvertretung ist in der Lage, ein einseitiges, vorgreifendes Handeln als ein in seinen Grundlagen auf Symmetrie beruhendes und damit im echten Sinne „kommunikatives Handeln ${ }^{13}$ zu beschreiben. Vom Ansatz her läßt sich mit Bonhoeffer ein ärztlicher Paternalismus vermeiden und lassen sich dennoch die tatsächlich vorhandenen Rollenunterschiede zwischen Arzt und Patient berücksichtigen. Aufgabe des verantwortlich Handelnden ist es, „die Verantwortlichkeit der ihm Anbefohlenen ins Bewußtsein zu erheben, zu stärken“. (S. 268)

Die Asymmetrie zwischen Arzt und Patient im Krankenhaus kann im folgenden Diagramm beschrieben werden ${ }^{14}$ :

$\begin{array}{ll}\text { Patient } & \text { Arzt, bzw. Pflegepersonal } \\ \text { Kranksein/Hinfälligkeit } & \text { Gesundheit } \\ \text { Hilflosigkeit } & \text { spezielle Kompetenz } \\ \text { existentielle Betroffenheit } & \text { Sachlichkeit/Routine (Distanz) } \\ \text { Dauer des Krankseins } & \text { Augenblick der Hilfe. }\end{array}$

Diese Asymmetrie ist unvermeidbar und verdient daher ehrlich beschrieben und nicht in wohlmeinender Absicht geleugnet zu werden. Es ist nicht so, als ob Arzt und Patient einander als zwei gleichermaßen unabhängige Individuen gegenüberträten: Vielmehr sind beide durch ihre unterschiedlichen Lebenssituationen existentiell jeweils unterschiedlich geprägt. Entscheidend ist nun, ob diese Asymmetrie von einer Wechselseitigkeit getragen wird. Im modernen Krankenhaus, so resümieren Gerta Scharffenorth und Klaus Müller, werden Patienten oft „in eine passive Rolle gedrängt und resignieren infolge mangelnder Kommunikation "15. Daher bestehe tendenziell die Gefahr, Patienten zu entmündigen, sei es, weil ihnen von ärztlicher Seite keine Selbständigkeit zugetraut wird, sei es, weil das - im Krankenhaus notwendige - System allseitiger Fürsorge regressiv wirkt und viele Patienten die eigene Mündigkeit nicht mehr anstreben.

Wenn medizinische Ethik zugleich als ärztliche Ethik und als Patientenethik beschrieben wird ${ }^{16}$, so ergibt sich aus Bonhoeffers Verantwortungsethik ein Vorschlag, wie beides aufeinander bezogen werden kann. Zwar hat bei Bonhoeffer die ärztliche Seite ein Übergewicht. Doch da Christus sich in der Menschwerdung mit der Krankheit als mit der verlorenen Welt identifiziert, hat ärztliches

\footnotetext{
${ }^{12}$ Vgl. Rendtorff, Ethik I, S. 103.

${ }^{13}$ So einen durch Habermas, Theorie, geprägten Begriff aufnehmend, Huber, Sozialethik, S. 144.

${ }^{14}$ Nach Raspe, Aufklärung; vgl. Scharffenorth/Müller, Patientenorientierung, S. 144, 215.

15 Vgl. ebenda, S. 40.

${ }^{16}$ Vgl. Rendtorff, Ethik II, S.215.
} 
Handeln sich an der Parteinahme Gottes für das Niedriggeachtete zu orientieren. Im Patienten als dem armen Bruder, der an die Tür klopft, begegnet der Arzt Christus. Daher kann von Bonhoeffer her Patientenorientierung ${ }^{17}$ als Aufgabe ärztlicher Ethik gefordert werden.

Bonhoeffers „Struktur verantwortlichen Lebens“ enthält als zweite Dimension die „Wirklichkeitsgemäßheit“, in welcher der Zusammenhang zwischen der (primären) Verantwortung für die Menschen und der (sekundären) Verantwortung für den „Bereich der Dinge“ unter dem Kriterium der „Sachgemäßheit“ verhandelt wird. (S. 269) „Sachgemäß“ ist dasjenige Verhalten zu den Dingen, welches „ihre ursprüngliche, wesenhafte und zielhafte Beziehung auf Gott und den Menschen im Auge behält" und zugleich das "Wesensgesetz" einer "Sache" (Logik, Staat, Familie, Aktiengesellschaft usw.) berücksichtigt. (S.269)

Wichtig an dieser Überlegung für die Medizin erscheint uns, daß sie geeignet ist, Sachgesichtspunkte in Beziehung zu personalem Handeln zu setzen: Sachgesichtspunkte dürfen und müssen berücksichtigt werden; jedoch verdient nur ein solches Handeln das Prädikat „sachgemäß“, bei dem um Christi willen das Handeln für den Menschen im Mittelpunkt bleibt. Bonhoeffer behauptet also nicht, $\mathrm{da} ß$ ein Sachwissen überflüssig ${ }^{18}$, daß etwa in der Medizin das Wissen des Patienten das des Arztes ersetzen könne. Wenn Bonhoeffer "Sachgemäßheit" und die Anerkennung von "Wesensgesetzen“ zum verantwortlichen Handeln zählt, ist hier Raum für eine medizinische Fachkenntnis, die auch den Gebrauch technischen Wissens einschließt. Jedoch ergibt sich das Handeln nie aus einer angeblichen "Eigengesetzlichkeit" von Lebensbereichen, weil immer die wesenhafte Ausrichtung aller Dinge auf Gott und den Menschen im Auge behalten werden muß.

Tatsächlich ist das Ersetzen zwischenmenschlicher Kommunikation ${ }^{19}$ durch Ergebnisse der Apparatemedizin gegenwärtig zu einem entscheidenden Problem geworden: „Der Patient wendet sich an den Arzt in Erwartung einer Antwort oder eines Rates. Dieser aber antwortet nicht direkt, sondern schaltet als Dritten einen Apparat ein, der seinerseits für ihn geeignete Information für den Patienten erhalten muß. Aus dem Dialog mit dem Apparat gewinnt der Arzt Kenntnisse, aufgrund derer er schließlich dem Patienten etwas mitteilt ${ }^{\text {“20. }}$ - „Der Patient scheint in eine Unzahl von Organsystemen zu zerfallen, diese in eine Unzahl von Krankheitsgebieten, für die es jeweils einen Spezialisten gibt, von der Patho-

\footnotetext{
17 Vgl. Scharffenorth/Müller, Patientenorientierung.

${ }^{18}$ Insofern halte ich es zumindest für unpräzise, wenn Honecker, Einführung, S. 331, zu Bonhoeffers Verantwortungsethik kritisch anmerkt, es sei eine offene Frage, ninwiefern Verantwortung überhaupt an Sachanforderungen zu messen ist". Diese Fehlinterpretation dient doch wohl vor allem Honeckers eigenem Programm, die Sozialethik habe eine „relative ,technische' Eigengesetzlichkeit" anzuerkennen; vgl. ebenda, S.324. Dagegen ist von Bonhoeffer her kritisch anzumerken, $\mathrm{da} ß$ alle Berücksichtigung von "Sachanforderungen“, welche dem Menschen schaden, von der Ethik nicht anzuerkennen ist (auch nicht relativ).

19 Vgl. Löning/Rehbein, Arzt-Patienten-Kommunikation.

${ }^{20}$ Scharffenorth/Müller, Patientenorientierung, S.74.
} 
logie bis zur Psychosomatik. Der Blick [des Arztes] muß sich verengen. Gleichzeitig nimmt das unsichere Gefühl des Arztes zu, seinen Patienten nicht ganz zu kennen, nur noch seinen Magen, sein Blutbild, seine Schilddrüse [. . .]. Der Patient wiederum lernt kurz einen Arzt fürs Herz, einen fürs Röntgen, einen fürs Blut, einen für Ultraschall usw. kennen“21. Die hier geschilderten Ambivalenzen des medizinischen Fortschritts sind faktisch vorhanden und weder durch schlichte Patentrezepte noch durch rückwärtsgewandte Utopien wie die „Neue Deutsche Heilkunde ${ }^{\text {"22 } 22}$ aus der Welt zu schaffen.

Im Sinne der Wirklichkeitsgemäßheit Bonhoeffers sind der Gebrauch technischer Apparate und die zunehmende Spezialisierung genau so weit und insofern legitim, wie dabei die „wesenhafte Ausrichtung aller Dinge auf Gott und den Menschen " im Auge behalten wird. Blinder Fortschrittsglaube ist damit ebenso ausgeschlossen wie naive Fortschrittsfeindlichkeit. Orientierung am Menschen mag angesichts der Eigendynamik, die der Gebrauch technischer Geräte, verstärkt durch ein sehr einseitiges Honorarsystem, entfalten kann ${ }^{23}$, ein hohes, gelegentlich wahrscheinlich sogar unerreichbares Ziel bilden. Dennoch wird ein am Menschen orientiertes ärztliches Handeln prinzipiell an dem Grundsatz festhalten müssen, ärztliche Leistungen nicht nach der Zahl von Verrichtungen (wie Operation, Bestrahlung, Medikation, Wundversorgung usw.), sondern an der positiven Beeinflussung des körperlichen und seelischen Gesamtzustandes, d.h. am Menschen selbst, zu bemessen ${ }^{24}$.

Die verbreitete Sichtweise, der Arzt würde "wertneutrale“ Informationen vermitteln, kann nicht gerechtfertigt werden, denn Informationen liegen nie anders als in gedeuteter Form vor. Bonhoeffers These, das "Wesensgesetz" einer Sache sei nicht einfach da, impliziert, daß es immer nur in anteilnehmendem Verhalten ermittelt werden kann. Daher gilt: „Ich habe [als Arzt oder Ärztin] nicht für den Patienten zu entscheiden, was für ihn gut ist, sondern die Vorteile und Nachteile möglicher Therapie mit ihm zu besprechen. Dabei sollte ich mit meiner Meinung und den Gründen dafür nicht hinter dem Berg halten. Weder die Erwartung, daß der Patient meine Meinung letztlich doch übernimmt, noch die Erwartung, daß in jedem Fall der Patient schon weiß, was für ihn richtig ist, führt zu einem sinnvollen Gespräch" ${ }^{\text {25 }}$.

Bonhoeffers „Struktur verantwortlichen Lebens" enthält als dritte Dimension die "Schuldübernahme“, das wohl ungewöhnlichste Element seiner Verantwortungsethik, bei dem zugleich die zeitgeschichtlichen Umstände am stärksten kenntlich sind. Schuldübernahme ist Bonhoeffer zufolge dort erforderlich, wo in einer schuldbehafteten Umwelt das starre Durchhalten eines an sich guten und sinnvollen ethischen Prinzips lieblos wäre: „Jesus will nicht auf Kosten der

\footnotetext{
${ }^{21}$ Themel, Entwicklungstendenzen, S. 51.

22 Siehe Einleitung.

${ }^{23}$ Vorschläge zu einem anderen Honorarsystem macht Breyer, Kalkulation.

${ }^{24} \mathrm{Vgl}$. Scharffenorth/Müller, Patientenorientierung, S.386f.

${ }^{25}$ Dörner/Plog, Irren, S. 165.
} 
Menschen als der einzig Vollkommene gelten, will nicht als der einzig Schuldlose auf die unter ihrer Schuld zugrundegehende Menschheit herabsehen, will nicht über den Trümmern einer an ihrer Schuld gescheiterten Menschheit irgendeine Idee eines neuen Menschen triumphieren lassen." (S. 275) Die Liebe Jesu, die dem schuldbehafteten Menschen gilt, läßt ihn nicht mit seiner Schuld allein, sondern nimmt an ihr Anteil. Auch jedes dem göttlichen Ruf antwortende menschliche Handeln hat partiell an der Schuldübernahme teil.

Handeln im Kontext einer schuldbehafteten Umwelt, im Kontext geschichtlich wirksamer und damit immer auch unvollkommener Strukturen, schließt die Bereitschaft zu Schuldübernahme ein. Niemand kann sich von der Schuld frei machen, sich damit entschuldigen, daß die Verhältnisse nicht so sind, wie er es gern hätte, oder damit, daß die Verhältnisse ihn zu diesem oder jenem Schritt genötigt hätten. Allerdings gibt es, Bonhoeffer zufolge, menschliche Zwangslagen, in denen der Mensch nur die Wahl zwischen zwei Übeln hat. Er soll dann nicht das „absolut Gute“ zu verwirklichen streben, welches gerade das Schlechteste sein kann, sondern in Selbstbescheidung das relativ Bessere dem relativ Schlechteren vorziehen ${ }^{26}$. Dies schließt aber die Bereitschaft zur Schulderkenntnis und Schuldübernahme ein: Das relativ Beste ist nicht das absolut Gute, sondern bleibt ein Übel. Wer meint, sich der Schuld entziehen zu können, wird der Selbsttäuschung erliegen und schließlich noch das relativ Schlechtere für das relativ Bessere halten. Verantwortliches ärztliches Handeln, das sich im Rahmen der Krankenhausstruktur, im Umgang mit einem vorgegebenen Honorarsystem oder den Vorgaben der Krankenkassen bewähren muß, wird nicht selten das eigene "prinzipielle" ethische Urteil in Frage gestellt finden. Sowohl die teilweise oder überwiegende Anpassung an das Vorgegebene wie auch dessen mögliche Revision ist Teil der Verantwortung.

Die ärztliche Verantwortung und die ärztliche Schuld werden nicht kleiner dadurch, daß sie ein unvermeidliches menschliches Verhängnis bleiben, in das die menschliche Existenz immer schon verwickelt ist. Sie werden einzig dadurch aufgehoben, wie Bonhoeffer ausführt, daß ein Mensch in der Auseinandersetzung mit der "notwendigen" Schuld und im Kampf mit seinem Gewissen unbedingt und völlig auf Gott und auf die Mitmenschen zurückverwiesen wird. „Das bedeutet, daß ich die Einheit mit mir selbst nur noch in der Hingabe meines Ich an Gott und die Menschen finden kann. Nicht ein Gesetz, sondern der lebendige Gott und der lebendige Mensch, wie er mir in Jesus Christus begegnet, ist Ursprung und Ziel meines Gewissens." (S. 279) Wer bewußt in die Schuldübernahme einwilligt, findet damit, paradoxerweise, zur Freiheit von der Schuld, nämlich von dem eigenen Bedürfnis nach Selbstrechtfertigung.

Dies bedeutet nun nicht Libertinismus: Denn es behält das ärztliche Gewissen sein Recht, ebenso wie die ärztliche Verpflichtung (das "Gesetz“, vgl. S.281ff.) gültig bleibt. Das Gewissen bewahrt dem Menschen, dem Arzt, seine Identität.

${ }^{26}$ Siehe S. 137. 
Eine Entscheidung kann nicht jenseits dieser Identität des Verantwortlichen liegen: „Es gibt Verantwortungen, die ich nicht zu tragen vermag, ohne daran zu zerbrechen [...] Zwar kann und soll die Tragkraft für verantwortliche Entscheidungen wachsen, zwar bedeutet jedes Versagen angesichts einer Verantwortung auch schon eine verantwortliche Entscheidung, dennoch bleibt im konkreten Fall der Ruf des Gewissens zur Einheit mit sich selbst in Jesus Christus unüberwindlich und es erklärt sich daraus die unendliche Mannigfaltigkeit verantwortlicher Entscheidungen." (S.282) Daraus folgt, daß eine Entscheidung von außen her kaum be- oder verurteilt werden kann. Das Element der Rechenschaftspflicht vor den Mitmenschen war in Bonhoeffers Verantwortungsethik nicht bestimmend. Es ist aber prinzipiell vorhanden, wie man den folgenden Sätzen entnehmen kann: „Wer in Verantwortung Schuld auf sich nimmt [...] der rechnet sich selbst und keinem anderen diese Schuld zu und steht für sie ein, verantwortet sie. [...] Vor den anderen Menschen rechtfertigt den Mann der freien Verantwortung die Not, vor sich selbst spricht ihn sein Gewissen frei, aber vor Gott hofft er auf Gnade." (S. 283)

Auch die ärztliche Verpflichtung - theologisch gesprochen: das Gesetz der Gottes- und Nächstenliebe - wird durch die Freiheit der Verantwortung nicht außer Kraft gesetzt, sondern nur jeweils suspendiert, insofern kein äußeres $\mathrm{Ge}$ setz das letzte Wort haben kann gegenüber der Verantwortlichkeit, die sich Gott verpflichtet weiß. Inneres Gewissen und äußere Verpflichtung bleiben also in Bonhoeffers Sicht unabdingbar, und die Spannung der verantwortlichen Entscheidung ist just auch die, nicht „in dem frevelnden Übermut seiner Macht, sondern in der Erkenntnis zu dieser Freiheit" zu handeln, einer Freiheit, die demütig ist im Wissen darum, daß sie im Gegenüber zu Gott auf Gnade angewiesen ist. (S. 283)

\section{Menschenwürde und Menschenrechte als Ansatz medizinischer Ethik}

\section{Menschenwürde im Widerspruch zum „Wert" menschlichen Lebens}

Bonhoeffer weist, so haben wir gesehen, dem Begriff der Menschenwürde eine zentrale Rolle bei der Beschreibung der Rechte leiblichen Lebens zu. Obwohl er teilweise die vernunftrechtliche Terminologie Kants benutzt, sieht er die Menschenwürde nicht im kategorischen Imperativ, sondern in Gottes gnädiger $\mathrm{Zu}$ wendung zum Menschen begründet. Die menschliche Würde ist nach Bonhoeffer im Rechtfertigungsgeschehen verankert: Der Mensch ist Mensch nicht kraft eigener ihm zukommender Eigenschaften oder von ihm zu erbringender Leistungen, sondern wird es erst durch Gottes gnädige Zusage.

Dieser Argumentationsgang liegt noch jenseits einer juristischen Kodifizierung der Menschenwürde. Heute hat sich die Situation insofern verändert, als der Be- 
griff Menschenwürde als verfassungsrechtlicher Terminus z.B. in der Präambel zur Charta der Vereinten Nationen vom 26.6. 1945, in der Allgemeinen Erklärung der Menschenrechte vom 10.12. 1948, in den beiden Menschenrechtspakten der Vereinten Nationen vom 16. Dezember 1966, in Art. 1 des Grundgesetzes der Bundesrepublik Deutschland und in den Präambeln einer Reihe anderer Verfassungen eine zentrale Stellung einnimmt ${ }^{27}$. Das sind nun Rechtstexte, die sich nicht auf die Rechtfertigungslehre beziehen, was durchaus sinnvoll erscheint. Ebenso wie eine christliche Ethik Aussagen darüber machen muß, worin sie die Menschenwürde begründet sieht, wenn sie das Adjektiv „christlich“ zu Recht tragen will, darf eine Verfassung oder eine völkerrechtlich verbindliche Erklärung der Menschenrechte eine christliche (oder eine vernunft-, naturrechtliche oder eine religiöse) Begründung für die Menschenwürde nicht geben, wenn sie die Religionsneutralität moderner Verfassungsstaaten wahren will ${ }^{28}$. Eine christliche Ethik soll gar nicht „religionsneutral“ sein, muß aber fähig sein, sich zur Religionsneutralität von Verfassungsstaaten in der Moderne konstruktiv in Beziehung zu setzen. Bonhoeffers Einbettung des Gedankens der Menschenwürde in die Dialektik von „Letztem“ und „Vorletztem“ ist dazu fähig, weil sie das Menschliche und Gute auch außerhalb des Raumes der Kirche zu würdigen vermag und doch die christliche Begründung im Gefälle vom Letzten zum Vorletzten gewahrt bleibt.

Bonhoeffers Gedankenansatz, daß der Sinn des Lebens nicht in irgendwelchen Werten besteht, sondern das Leben selbst von Gott gutgeheißen wird, impliziert, daß das menschliche Leben eine Würde besitzt und als "über allen Preis erhaben“ geachtet werden muß $\beta^{29}$. Dieser Grundsatz kann gegen die vermeintlich neutralen Wissenschaftsbegriffe der Psychiatrie wie "hochwertig“ und „minderwertig“ ausgespielt werden. Doch wichtiger als diese zeitbedingte Terminologie ist der ethische Grundsatz, daß die Würde menschlichen Lebens nie nach dem „Wert" von Gesundheit oder Krankheit bemessen werden darf, selbst dann nicht, wenn die Krankheit als genetische Krankheit die menschliche Natur, das menschliche "Wesen" als solches betreffen sollte. Schon gar nicht darf die Ethik das menschliche Leben und die menschliche Gesundheit als zwei „Werte“ beschreiben, die im Konfliktfall je nach persönlicher Präferenz unterschiedlich abgewogen werden könnten. Vielmehr sind sämtliche Handlungsweisen daran zu messen, ob sie die Würde menschlichen Lebens verletzen.

Dieser an der Würde menschlichen Lebens orientierte Ansatz profiliert sich dann, wenn er mit denjenigen ethischen Modellen konfrontiert wird, die in unterschiedlicher Weise den "Wert" menschlichen Lebens in den Mittelpunkt stellen. Für eine solche „Ethik der Bewertung" lassen sich zwei unterschiedliche Gedankenansätze herausarbeiten: Entweder wird menschliches Leben nach dem

\footnotetext{
$27 \mathrm{Vgl}$. Charta und Human Rights.

$28 \mathrm{Vgl}$. Huber, Menschenrechte, Menschenwürde, S. 581.

${ }^{29}$ So die klassische Formulierung Kants, siehe S.146 Anm.71.
} 
Wert bemessen, den die Gesellschaft ihm zuschreibt. Oder aber das Leben wird nach dem Wert bemessen, den es nach der Meinung des Betroffenen hat, bzw. nach dem Wert klassifiziert, den es im Urteil Dritter für den Betroffenen haben soll. Im Rahmen des ersten Denkmodells ist „Minderwertigkeit" im Falle von Arbeitsunfähigkeit oder Anstaltspflegebedürftigkeit gegeben. Dieses Denkmodell hat Foster Kennedy radikal und konsequent zu Ende gedacht hat, indem er „Euthanasie“-Forderungen gegen alle diejenigen richtete, welche den Normen einer industriellen Leistungsgesellschaft nicht genügen, vor allem gegen geistig Behinderte ${ }^{30}$. Im Rahmen des zweiten Denkmodells wird körperliches oder seelisches Leid zum Kriterium für die „Minderwertigkeit“. In dieser Hinsicht konsequent ist Eugen Bleuler, indem er „Euthanasie“-Forderungen gegen alle diejenigen richtet, welche die gestellte Norm, glücklich sein zu müssen, nicht erfüllen können: gegen Menschen mit melancholischer Depression oder Schizophrenie ${ }^{31}$.

Die nationalsozialistische Gesundheitspolitik war durch eine Kombination beider Gedankenansätze, wohl mit besonderem Akzent auf dem erstgenannten, gekennzeichnet $^{32}$. Heutige Überlegungen, vor allem im Kontext utilitaristischer Ethiken, akzentuieren stärker das zweite Kriterium. Doch kann auch der heute anzutreffende scheinbar soziale Ansatz eines Handelns zum Besten des Patienten eigenartige Konsequenzen beinhalten: Wem es nach Auffassung Dritter nicht gut genug geht, der oder die hat damit auch das Recht auf Leben insgesamt verwirkt. Oder härter noch, da sich hier die Position einer Bestimmung durch Dritte völlig entzieht: Wer wegen akuter Depressionen nicht mehr leben will, der oder die bekommt diesen Wunsch von ärztlicher Seite augenblicklich erfüllt ${ }^{33}$.

In etwas anderer Weise gilt dies für den Präferenzutilitarimus Peter Singers, den man auch eine „Ethik der Interessen " ${ }^{34}$ nennen könnte. Hier wird davon ausgegangen, daß es in der Situation neuzeitlichen Pluralismus keine übergeordneten Prinzipien gebe, anhand derer ethische Konfliktfragen entschieden werden könnten. Medizinethische Urteile haben sich ausschließlich an den Interessen der beteiligten Personen zu orientieren, wobei gefordert wird, daß möglichst viele Präferenzen möglichst vieler beteiligter Personen Berücksichtigung finden. Entscheidend ist nun, daß derjenige, der seine Interessen nicht artikulieren kann, weil er keine Person ist (und als Person wird nur anerkannt, wer über die Merkmale Selbstbewußtsein und Rationalität verfügt), keinen eigenständigen Platz besitzt. Faktisch wird damit wird der Begriff Mensch durch den der Person ersetzt und der Begriff Person über Merkmale definiert, über die die meisten Menschen (gemeinsam mit höheren Säugetieren) verfügen, andere Menschen aber (geistig

\footnotetext{
${ }^{30}$ Siehe S. 24.

31 Siehe S.24.

${ }^{32}$ Siehe S. $54 \mathrm{f}$.

${ }^{33}$ Dies waren extreme Konsequenzen einer heute kaum noch diskutierten Anti-Psychiatrie; vgl. Szasz, Geisteskrankheit, S.295; kritisch dazu Müller-Hegemann, Medizinische Psychotherapie, S. 84 .

${ }^{34}$ Vgl. Huber, Grenzen, und die Replik von Kupatt, Wie weit?
} 
Schwerstbehinderte und Säuglinge) nicht. Nicht-Personen besitzen keine Individualität und können nach Singer „ausgewechselt", behinderte oder kranke Babys (er denkt an die Diagnosen spina bifida, Hämophilie und Down-Syndrom) umgebracht und durch die Geburt eines gesunden Kindes „ersetzt" werden ${ }^{35}$.

An der extremen Position Singers läßt sich erkennen, wohin eine „Ethik der Interessen“ als Denkansatz führen kann, nämlich dazu, daß die Menschenwürde durch eine „Personenwürde“ ersetzt wird und man denjenigen Menschen, die ihre Interessen nicht artikulieren können (sog. „Nicht-Personen“), die Menschenwürde de facto abspricht. Konsequenterweise kann man dann sogar einen Intelligenzquotienten von IQ 20 verlangen, um jemanden als Person anzuerken$n^{36}{ }^{36}$. Doch erscheint die einseitige Bevorzugung rationalen gegenüber nicht-rationalem menschlichen Leben "rational“ keineswegs besser begründet als der durch die Rechtfertigungslehre gebotene Verzicht auf die Unterscheidung zwischen Personen und Nicht-Personen. Alle Menschen kennen vorübergehende Phasen der Nicht-Rationalität und des Nicht-Bewußtseins zumindest aus dem Schlaf, und Bonhoeffers Ansatz beim leiblichen Leben kann erklären, wieso der bewußtseinslose Schlaf als vollgültiger Bestandteil menschlichen Lebens und nicht als ein irgendwie defizienter Modus des Menschseins interpretiert werden muß.

Nun sind auch in einer Ethik der Würde menschliche Interessen keineswegs irrelevant. Bonhoeffer zufolge ist ja das menschliche Glück ethisch nicht unerheblich: Streben nach Glück wird sogar als ein Menschenrecht bestimmt. Doch zugleich sieht Bonhoeffer auch das unglückliche, das körperlich oder seelisch leidende menschliche Leben als vollgültiges, ja sogar als ein christlich besonders qualifiziertes Leben an, dem die Menschenwürde nicht abgesprochen, ein Minderwert nicht nachgesagt werden darf. Er steht damit einer „Glorifizierung des Leidens ${ }^{437}$ nicht minder fern wie einem moralisierenden Zwang zum eigenen Glück.

${ }^{35}$ Vgl. Singer, Praktische Ethik, S. 106, 131, 181, 183, 185. Zustimmung erntete Singer quer durch die politischen Lager, im konservativen Deutschen Ärzteblatt, vgl. Kuhse, Warum, in einem von 31 Philosophinnen und Philosophen (darunter Theunissen und Tugendhat) unterzeichneten Appell in der alternativen tageszeitung vom 6.1. 1990, in der feministischen Frauenzeitschrift Emma vom März/April 1944, vgl. NN, Affentheater, S.107ff., in den orthodox marxistischen Ketzerbriefen, vgl. Heft 40 (1983), S. 64-77, und, zumindest mehrheitlich, in einer Artikelserie der liberalen Zeit von Juni bis September 1989.

${ }^{36}$ So der Vertreter einer radikalen Situationsethik, Fletscher, Indicators of Humanhood: A tentative profile of Man, in: The Hastings Center Report, Vol. 2 No. 5, S.1, nach Bayertz, GenEthik, S. 179.

${ }^{37}$ So der unberechtigte Vorwurf von Kurt Bayertz, GenEthik, S. 213, gegen Eibach, Experimentierfeld, S.148. Der angeblichen "Glorifizierung des Leidens" im Christentum stellt Bayertz das ethische Postulat entgegen, Leid "mit allen Mitteln zu bekämpfen“. Dazu gehören auch die durch die „Errungenschaften der Gen- und Reproduktionstechnologie eröffneten Optionen zu einer bewußten und gezielten Steuerung der menschlichen Fortpflanzung", wozu gentechnische Eingriffe in die Keimbahn eingesetzt werden dürfen, nicht nur zu therapeutischen Zwecken (Gentherapie'), sondern auch zu sonstigen „Verbesserungen“ des Erbgutes; vgl. ebenda, S.289f. Dieses Plädoyer scheint dem Autor mit einer Kritik eugenischer Bestrebungen im Nationalsozialismus vereinbar zu sein, vgl. Weingart/Kroll/Bayertz, Rasse. 
Die Notwendigkeit dieser theologisch reflektierten Dialektik zwischen einem Recht auf Streben nach Glück und einer besonderen Qualifizierung leidvollen Lebens kommt deutlicher zum Tragen, wenn wir sie im Vergleich zu denjenigen sozialmedizinischen Überlegungen betrachten, welche das Anliegen verfolgen, bei der statistischen Auswertung von Therapien neben der Erfolgsquote (Heilung/Nicht-Heilung) auch die „Lebensqualität" einzubeziehen.

Eine Bemessung der Lebensqualität zielt darauf, die Bedürfnisse des Behandelten einzubeziehen. So erscheint es sicherlich sinnvoll, dem Patienten Fragen zu stellen wie „Müssen Sie den größten Teil Ihres Tages im Bett oder Sessel verbringen?", „Können Sie sich allein versorgen?“, „Haben Sie Schmerzen?“, „Sind Sie müde?", „Fühlen Sie sich niedergeschlagen?" etc., und diese Fragen bei der Beurteilung verschiedener therapeutischer Methoden statistisch auszuwerten. Jedoch wird dabei nicht der "Wert" menschlichen Lebens ermittelt und schon gar nicht eine Entscheidung darüber getroffen, welches Leben „lebenswert“ und welches „lebensunwert" $\operatorname{se}^{i 38}$, sondern nur das Ausmaß bestimmter Bedürfnisbefriedigungen festgehalten.

Die beiden genannten Arten der Bewertung menschlichen Lebens (Wert für die Gesellschaft/Wert für das Individuum selbst) tauchen abermals in der Diskussion um die Frage auf, ob eine Messung von Lebensqualität sich an „objektiven“ oder an "subjektiven“ Kriterien zu orientieren habe. Als „objektive“ Kriterien gelten diejenigen, die für die Mitwelt relevant und beobachtbar sind, von der Bettlägrigkeit bis zur Fähigkeit, dem eigenen Beruf nachzugehen. Doch ist dieses Kriterium durchaus einseitig. Beispielsweise muß die „objektiv“ beobachtbare Einbuße an Lebensqualität eine krebskranke Patientin nicht daran hindern, den auf wackligen Beinen verbrachten Ski-Urlaub zwischen zwei Chemotherapien „subjektiv" als den glücklichsten ihres Lebens anzusehen ${ }^{39}$. Schon gar nicht dürfen die mit einer geistigen Behinderung verbundenen „objektiven“ Einschränkungen der Lebensqualität von Dritten als ein besonderes Unglück gedeutet werden. Dies geschieht aber dort, wo man meint, daß „Bewußtsein, Vernünftigkeit, Verantwortlichkeit" die Bedingung der Realisierung menschlichen Lebens und menschlicher Lebensqualität seien, daß die „rationalen Möglichkeiten der Güterabwägung in bezug auf lebensstilrelevante Optionen in der individuellen Ausgestaltung von Lebensqualität“ überhaupt herangezogen werden dürften ${ }^{40}$.

\footnotetext{
${ }^{38}$ So aber Schara, Was bedeutet?, S. 5 .

${ }^{39}$ Vgl. aus der Sicht einer Betroffenen Suritsch-Bauer, Lebensqualität, S. 40.

${ }^{40}$ Vgl. Sass, Lebensqualität, S.13. Zwar wird dieser Ansatz gleich wieder eingeschränkt, wenn Sass erklärt, dies beinhalte „in keinem Fall den Ausschluß des Geistigbehinderten aus der mitmenschlichen Gemeinschaft und Solidarität“. Vgl. ebenda. Gleichwohl erscheint befremdlich, daß diese Einschränkung überhaupt erforderlich ist. Geistig Behinderte, die den ganzen Tag ,in einem Zimmer sitzen, Kuscheltiere an die Wand schmeißen, ab und an ein paar Stunden im Garten verbringen“, werden wohl bei den „rationalen Möglichkeiten der Güterabwägung in bezug auf lebensstilrelevante Optionen in der Ausgestaltung von Lebensqualität" nicht ganz mithalten können gegenüber einem arbeitenden „nicht-rauchenden und joggenden Familienvater"; so Tolmein, Wann?, S. $149 \mathrm{f}$.
} 
"Subjektive" Lebensqualitätsdefinitionen dagegen beziehen sich auf das momentane Empfinden, von körperlichen Schmerzen bis hin zur Lebensfreude. Auch dieser Maßstab ist einseitig, da die menschlichen Gefühle, besonders in Krankenhäusern, starken Schwankungen ausgesetzt sind und zudem oft von Medizin-fremden Kriterien abhängen, von der Atmosphäre im Krankenhaus zum Beispiel oder von der Häufigkeit von Krankenbesuchen. Der Versuch, diesem Mangel zu begegnen, indem Lebensqualität als die Summe von Tageslaunen definiert, Lebensqualität und Lebensdauer durch die Berechnung von "quality adjusted life years" (QALY) ${ }^{41}$ mathematisch indiziert werden, macht schließlich aus dem menschlichen Leben eine bilanzierbare Gewinn- und Verlustrechnung, dessen Wert durch die Summe aller konsumierten Glücks-Zeit-Einheiten bestimmt wird. Zwar handelt es sich tatsächlich um ernste Fragen, wenn aufgrund einer schweren Krankheit Lebensjahre gegen Lebensqualität abgewogen werden müssen, ob beispielsweise bei Krebs eine Chemotherapie, eine Brustamputation oder ein künstlicher Darmausgang für eine gewisse, statistisch errechenbare Verbesserung der Heilungschancen oder eine Lebensverlängerung sinnvoll erscheinen. Doch können solche wirklich ernsten und existentiellen Fragen nicht durch entmündigende statistische Vermessungen menschlichen Glücks beantwortet werden, sondern nur (nach ärztlicher Beratung) von den Betroffenen selbst. Hier gar den Wert menschlichen Lebens in einer Geldsumme bemessen zu wol$\mathrm{len}^{42}$, um sich durch solche Berechnungen berechtigt $\mathrm{zu}$ sehen, eine medizinische Behandlung dort abzubrechen, wo die aufzuwendenden Kosten diese Geldsumme übersteigen ${ }^{43}$, braucht wohl nicht weiter diskutiert zu werden. Zu Recht erklären die Kirchen: „Keiner hat über den Wert oder Unwert eines anderen menschlichen Lebens zu befinden - selbst nicht über das eigene ${ }^{\text {"44 }}$.

Eine auf der Menschenwürde beharrende Ethik muß also Bewertungen menschlichen Lebens in Fremdperspektive und in Eigenperspektive ausschließen. Wohl ist im Rahmen einer Ethik der Würde die Berücksichtigung menschlicher Interessen nicht ausgeschlossen, wie sie auch mit einem Recht auf Streben nach Glück vereinbar ist. Daher können in der Medizin „objektive“ oder „subjektive" Faktoren der Lebensqualität sehr wohl Berücksichtigung finden, sofern dabei die Relativität derartiger Definitionen bewußt bleibt und keine Aussage über einen angeblichen "Wert" menschlichen Lebens getroffen wird. Eine Ethik der Würde verweigert sich aber jeder Instrumentalisierung menschlichen Lebens zu einem fremden $Z$ weck.

\footnotetext{
${ }^{41}$ Vgl. Weinstein, Clinical Decision; verhaltene Kritik übt Klingler, Statistische Auswertung, S. 157.

${ }^{42}$ So Meggle nach Tolmein, Wann, S. 156.

${ }^{43} \mathrm{Vgl}$. Engelhardt, Zielkonflikte, S. 35.

${ }^{44}$ Vgl. Gott ist ein Freund des Lebens, S. 107.
} 


\section{Menschenrechte als Ansatz medizinischer Ethik}

„Beim Thema Menschenrechte haben die Menschen seit Jahrhunderten über Zielsetzung und Orientierung des Menschseins nachgedacht. Es mag erstaunen, damit in einer medizinischen Ethik einzusetzen. Aber die Menschenrechte enthalten seit jeher ein solches lebensförderndes, kritisches und emanzipatorisches Potential. Ich mache also den fast banalen Vorschlag, der Arzt solle sich in seinem Handeln zunächst schlicht an wesentlichen Menschenrechten orientie-

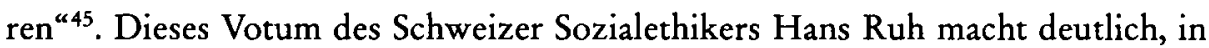
welcher Weise die Menschenrechte als Ansatz medizinischer Ethik ein halbes Jahrhundert nach Bonhoeffer diskutiert werden. Letzterer hat auf die Menschenrechte primär zur Abwehr ungeheurer Verbrechen zurückgegriffen. Unsere Gegenwart ist durch nichts Vergleichbares gekennzeichnet. Dennoch besitzen die Menschenrechte auch in der Gegenwart die Funktion, Verbrechen abzuwehren - sei es auch nur präventiv in der Form von Warnungen und Verboten. Gleich ob man die Erprobung neuer Medikamente und Therapien, Fragen medizinischer Ethik in der sog. „Dritten Welt“ oder die neuesten Anwendungen der Gentechnik diskutiert: Ethisch verantwortliches Handeln kann Überlegungen zu den Menschenrechten nicht vernachlässigen.

In der multikulturellen Gesellschaft bilden die Menschenrechte eine entscheidende Regelungsnorm für interkulturelle oder interreligiöse Konflikte: Sofern Ärztinnen und Ärzte, Patientinnen, Patienten und Pflegepersonal unterschiedlicher religiöser oder weltanschaulicher Prägung und Herkunft sich begegnen, kann es fraglich erscheinen, wessen Tradition sich im Zweifelsfall durchsetzt. Besonders bei Fragen, die in unterschiedlichen Traditionen unterschiedlich beantwortet werden (z.B. Verhütung, In-vitro-Fertilisation, Schwangerschaftsabbruch, Organtransplantation, Bluttransfusion) kann dies zum Problem werden nicht nur in konfessionell geführten Krankenhäusern. Hier bieten sich die Menschenrechte als ein Bezugsrahmen an, der Kriterien zur Lösung derartiger Konflikte vorgibt. Die Menschenrechte können dabei am ehesten als diejenigen Werte beurteilt werden, auf die Menschen unterschiedlicher Tradition sich geeinigt haben und einigen können.

Für die medizinische Ethik sind eine Reihe relevanter, international anerkannter materialer Bestimmungen entstanden: Schon anläßlich des Nürnberger Ärzteprozesses kam es zu Resolutionen über Versuche am Menschen; später bildeten die Helsinki-Deklaration von 1964 und ihre Neufassung von Tokio 1975 wichtige Meilensteine für die Menschenrechte als Thema medizinischer Ethik ${ }^{46}$. Das Council for International Organization of Medical Science erstellt Resolutionen, zu denen auch die Christian Medical Commission des Ökumenischen Rates der Kirchen Stellung nimmt. Diese werden von den relevanten ärztlichen Standesor-

\footnotetext{
${ }^{45}$ Ruh, Argument Ethik, S. 133.

${ }^{46}$ Literaturangaben in: Human Rights in Health.
} 
ganisationen der meisten Länder akzeptiert. Ein Problem liegt allerdings nach wie vor darin, daß die Anerkennung teilweise nur auf dem Papier erfolgt bzw. im Konfliktfall fast immer dem nationalen Recht die entscheidende Durchsetzungskraft zukommt - selbst dann, wenn es explizit einzelnen international anerkannten Bestimmungen widerspricht. Hierbei handelt es sich jedoch, wie zumeist bei Menschenrechtsfragen, weniger um eine Frage der Rechtsbegründung als um ein Vollzugsdefizit. Dennoch ist die Respektierung der Menschenrechte der Beliebigkeit entzogen. Jede institutionelle oder personale Beziehung zwischen Menschen muß die Existenz von Menschenrechten als im voraus gegeben anerkennen. Die durch alle UNO-Mitgliedsstaaten anerkannten Menschenrechtserklärungen finden dem Grundsatz nach, trotz bedauerlich häufiger Mißachtung, so breite internationale Anerkennung, daß sie am ehesten als so etwas wie das „Weltethos ${ }^{\text {“47 }}$ angesehen werden können.

Nicht alle Fragen medizinischer Ethik können direkt durch den Hinweis auf materiale Menschenrechtsnormen "gelöst" werden. Ein an den Menschenrechten orientiertes medizinisches Ethos kann jedoch auch dort Überlegungen für die ethische Urteilsbildung geltend machen, wo explizite juristische Normen nicht greifen. Man wird hier nicht danach streben, die Grenzen dessen, was verfassungsrechtlich gerade noch zulässig ist, auszuschöpfen, sondern seine ethischen Handlungen an der zentralen Idee des Menschenrechtsgedankens, der Achtung und Respektierung der Menschenwürde, bemessen. Die Menschenrechte können als "Leitsätze zur ethischen Orientierung“ den „Problemhorizont“ auch für solche Fragen abstecken, zu denen explizite Menschenrechtsnormen fehlen ${ }^{48}$. Wo die Medizin sich schneller entwickelt, als Rechtsinstitutionen reagieren können, erscheint dieser Grundsatz besonders wichtig. So konnte schon Dietrich Bonhoeffer den Grundgedanken der Menschenrechte gegen die Zwangssterilisation ausspielen, obwohl es keine international anerkannte Menschenrechtsnorm gab, welche die Zwangssterilisation explizit ausgeschlossen hätte. Ähnlich haben wir die (von Karl Bonhoeffer als Eingriff in das Arztgeheimnis verweigerte) Anzeigepflicht in Kombination mit der Zwangssterilisation als ein dem Grundgedanken der Menschenrechte widersprechendes Kennzeichen eines totalitären Überwachungsstaates und als schweren Eingriff in die Arzt-Patient-Beziehung beurteilt, obwohl eine ärztliche Anzeigepflicht bei Infektions- und Geschlechtskrankheiten damals wie heute in vielen Ländern zulässig war und keine Menschenrechtsnorm eine ärztliche Anzeigepflicht grundsätzlich ausschließt ${ }^{49}$.

Bonhoeffer bringt in den allgemeinen Menschenrechtsdiskurs ein Verständnis der Menschenrechte ein, welches diese von der christlichen Tradition her in einer ganz bestimmten Hinsicht qualifiziert. Im folgenden fassen wir den spezifischen Beitrag Dietrich Bonhoeffers zur Frage der Menschenrechte im Kontext heuti-

\footnotetext{
${ }^{47}$ Vgl. Huber, Menschenrechte, Menschenwürde, S. 577-602.

${ }^{48}$ Vgl. Ritschl, Menschenrechte, S. 262.

${ }^{49}$ Siehe S. $27 \mathrm{f}$.
} 
ger medizinischer Ethik unter drei Gesichtspunkten zusammen. Dabei wird deutlich, daß Bonhoeffers weitreichende Überlegungen teilweise als Bestätigung, zum Teil aber auch als Infragestellung der gegenwärtigen Diskussion zu lesen sind.

a) Bonhoeffers Verständnis von Krankheit und Gesundheit vermag zur Nüchternheit im Umgang mit den Menschenrechten als Thema medizinischer Ethik zu verhelfen und übertriebene Erwartungen an die Medizin zu vermeiden. So erscheint es von Bonhoeffer her fragwürdig, wenn in der Präambel der Weltgesundheitsorganisation (WHO) der Vereinten Nationen ein Recht auf Gesundheit postuliert und Gesundheit als Zustand des vollständigen körperlichen, geistigen und sozialen Wohlbefindens (und nicht nur des Freiseins von Krankheit und Gebrechen) definiert wird ${ }^{50}$. Damit werden an Medizin und Rechtsordnung Erwartungen gerichtet, die beide nur enttäuschen können. Ein „Zustand vollständigen körperlichen, geistigen und sozialen Wohlbefindens" kann nach christlichem Verständnis durch Menschen nicht geschaffen werden. Krankheit ist und bleibt, so führt Bonhoeffer aus, ein Teil der menschlichen Existenz ${ }^{51}$.

So kann der Verzicht auf ein übertriebenes Vertrauen in ein irdisches Gesundheits-Paradies zu einem gelasseneren Umgang mit dem Thema Krankheit führen; und man wird angesichts der faktisch vorhandenen Leiden auch umgekehrt nicht in übertriebenen resignativen Pessimismus verfallen. Beide Tendenzen, übertriebener Optimismus und übertriebener Pessimismus, konnten auch anhand der nationalsozialistischen Rassenhygiene beobachtet werden: Auf der einen Seite wurden übertriebene Hoffnungen in den „Erfolg“ der Zwangssterilisation bei der Ausrottung von Erbkrankheiten gesetzt ${ }^{52}$. Auf der anderen Seite tendierte ein übertriebener Pessimismus der Psychiatrie dazu, Menschen als „unheilbar" auszugrenzen und der "Euthanasie“-Aktion zu übergeben. Es scheint, daß übertriebener Optimismus und übertriebener Pessimismus in bezug auf die Möglichkeiten der Medizin zwei einander nur scheinbar ausschließende Spielarten einer unrealistischen Sichtweise der Medizin bildeten, zu der Karl Bonhoeffers Eintreten für eine "mittlere Linie des Fortschritts ${ }^{\alpha 3}$ eine echte Alternative bot. Dementsprechend ist zu fordern, daß auch der Rechtsbegriff nicht mit einem unerreichbaren Ideal aufgeladen wird: nicht ein Ideal, sondern die vor Gericht einklagbare Rechtsvorschrift ist das Ziel. Ähnlich wie das „Recht auf Leben" nicht etwa einen Rechtsanspruch auf Unsterblichkeit beinhaltet, sondern dem Staat lediglich abverlangt, unschuldiges Leben nicht willkürlich zu töten, kann ein „Recht auf Gesundheit“ als ein Rechtsanspruch auf medizinische Versorgung - nicht mehr, aber auch nicht weniger - interpretiert werden.

b) Dietrich Bonhoeffer bietet ein an der Sozialität orientiertes, nicht ein individualistisches Verständnis der Menschenrechte an. Wohl hat er die das Individu-

\footnotetext{
50 Nach Bundesgesetzblatt 1974 II, S. 45.

51 Siehe S. 129.

52 Siehe S. $42 \mathrm{f}$.

53 Siehe S.60.
} 
um schützenden Elemente der Menschenrechtstheorie integriert, indem auch bei ihm Menschenrechte zur Abwehr staatlicher Zwangseingriffe dienen. Jedoch läßt er sich nicht auf eine individualistische Anthropologie ein und beschränkt die Menschenrechte daher nicht auf ihre Abwehrfunktion; er bietet ein Menschenrechtsverständnis an, das die Rechte des einzelnen nicht einseitig egoistisch interpretiert, wie es in liberalen kapitalistischen Verhältnissen tendenziell immer eine Gefahr gewesen ist ${ }^{54}$. Ein individualistisches Verständnis der Menschenrechte ist zweifelsohne die nach 1945 in Deutschland vorherrschende Interpretation. Dennoch werden bis heute philosophische und theologische Gegenkonzepte vorgetragen. So denkt Jürgen Habermas private und öffentliche Autonomie, Menschenrechte und Volkssouveränität, mit Hilfe des Diskursprinzips so, daß sie

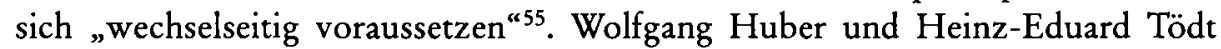
haben geltend gemacht, daß die Menschenrechte nicht allein durch Freiheitsrechte, sondern durch drei Sachmomente, nämlich Freiheit, Gleichheit und Teilhabe, gekennzeichnet seien, und formulieren die "hermeneutische Regel“, wonach ein Sachmoment nur unter Berücksichtigung der Korrelation aller drei untereinander verstanden werden könne ${ }^{56}$. Beide hier nicht im einzelnen zu diskutierenden Ansätze haben die Defizite eines auf die Freiheitsrechte des einzelnen reduzierten Menschenrechtsverständnisses deutlich gemacht.

Dies gilt auch für die medizinische Ethik. Werden etwa Selbstbestimmung, Mitsprache, Freiheit und Würde der Person als die zentralen Menschenrechtsbegriffe medizinischer Ethik herausgestellt ${ }^{57}$, so werden die Sozialbezüge, innerhalb derer Individuen ihre individuellen Entscheidungen treffen, von der Krankenversicherung bis zu sozialen Zwangslagen, unterbewertet. So ist z. B. die Reihenuntersuchung auf genetische Krankheiten durch einen Arbeitgeber vor Einstellung mit einem individualistischen Menschenrechtsverständnis so lange vereinbar, wie der Arbeitsuchende seine persönliche Einwilligung zu dieser Untersuchung erteilt. Doch liegt es auf der Hand, daß die Wahlmöglichkeit, sich genetisch untersuchen zu lassen oder keinen Arbeitsplatz zu finden, für das Individuum sehr beschränkt ist, wie umgekehrt bei konsequenter Reihenuntersuchung genetisch kranke Menschen äußerst schlechte Chancen auf einen Arbeitsplatz besitzen. Ein Menschenrechtsverständnis, welches Individualität und Sozialität des Menschen als ursprünglich gegeben und einander gleichwertig anerkennt, wird die Zustimmung des einzelnen nicht für weniger wichtig halten als die gesellschaftlichen Bezüge, innerhalb derer einzelne ihre Zustimmung erteilen.

Ein Rechtsverständnis, welches Recht und Liebe weder trennt noch identifi$z_{i e r t^{58}}$, beschreibt Recht nicht primär als die gegen andere zu verteidigenden Rechte des einzelnen, sondern ist offen für ein solidarisches Handeln: Weil ich

\footnotetext{
${ }^{54} \mathrm{Vgl}$. Macpherson, Politische Theorie.

${ }^{55}$ Habermas, Faktizität, S. 112.

${ }^{56}$ Huber/Tödt, Menschenrechte, S. 88-96.

${ }^{57}$ Vgl. Ruh, Argument Ethik, S. 135.

${ }^{58}$ Siehe S. 149.
} 
mich mit anderen fundamental verbunden weiß, kann ich auch für die Rechte anderer eintreten. Dieser Gesichtspunkt kann kritisch gegen alle Aufweichungen des Solidaritätsprinzipes zur Geltung gebracht werden, wie sie z. B. bei der Krankenversicherung der Gegenwart deutlich werden, wo "gute Risiken“ durch private Versicherungsträger individualisiert werden, während "schlechte Risiken“, die durchschnittlich mit höheren Kosten belastete Krankenversicherung von Arbeitslosen oder Sozialhilfeempfängern, den gesetzlichen Kassen überlassen werden.

c) Als der wichtigste Gesichtspunkt jedoch, den Bonhoeffer in den allgemeinen Menschenrechtsdiskurs der Gegenwart einbringt, erscheint seine Überlegung zur Verknüpfung von Leiblichkeit und Menschenwürde. Bonhoeffers Verständnis der Menschenwürde als einer Würde, welche zunächst dem menschlichen Leib, dann aber auch dem menschlichen Geist zukommt, bringt zum Ausdruck, daß nicht nur der menschliche Geist, sondern auch der Leib in erster Linie kein Mittel zum Zweck sein darf. Damit variiert Bonhoeffer den Gedanken Kants, daß kein Mensch gegenüber einem anderen einen vollständigen Verfügungsanspruch erheben darf. Das heißt hinsichtlich menschlicher Leiblichkeit: Auch der Leib des Menschen ist unersetzlich und kann durch einen anderen nicht ersetzt werden. Es ist nicht so, daß die menschliche Seele (etwa als ein Ding an sich im Sinne Kants) unberührt davon bleiben könnte, wenn der Körper mißhandelt oder ihm Gewalt angetan würde. Es ist vielmehr so, daß alle meinem Körper von anderen angetane Gewalt mir angetane Gewalt ist ${ }^{59}$. Zur Begründung verweist Bonhoeffer in deutlich antispiritualistischer Polemik darauf, daß der Mensch nach biblischem Verständnis keinen Leib hat, sondern Leib ist.

"Im Dialog mit der Medizin wirkt sich die ,Leibfremdheit" der Theologie, die in der ökumenischen Diskussion und in feministischer Theologie kritisiert wird, immer wieder als schwerwiegender Mangel aus", resumieren Gerta Scharffenorth und Klaus Müller ${ }^{60}$. Doch ist diese im Verlauf der Kirchengeschichte immer wieder zu beobachtende Leibfremdheit ${ }^{61}$ historisch auf die Verbindung des Christentums mit platonischem bzw. neoplatonischem Gedankengut und nicht auf biblisches Gedankengut zurückzuführen. Biblische Schriften vermitteln sehr konkrete Erfahrungen von Menschen, die mit ,Leib und Seele in Not gerieten und sich nach Heilung sehnten. Der Gedanke der Auferstehung des Fleisches, der Victor von Weizsäcker zufolge neben der Liebe den wichtigsten Beitrag des Christentums zur Medizin darstell $t^{62}$, ist derjenige dogmatische Topos, an dem klassischerweise die Auseinandersetzung mit der platonischen Seelenlehre geführt wurde.

\footnotetext{
59 Ähnlich Hegel, Rechtsphilosophie $\$ 48, S .111 \mathrm{f}$.

${ }^{60}$ Scharffenorth/Müller, Patientenorientierung, S. 35.

${ }^{61} \mathrm{Vgl}$. Shrey, Leib/Leiblichkeit, S. 638-643.

${ }^{62}$ Vgl. Weizsäcker, Frage, S. 229.
} 
Bonhoeffer lehnt den platonischen Leib-Seele-Dualismus $\mathrm{ab}^{63}$, ohne deswegen einem Monismus zu verfallen: $\mathrm{Er}$ argumentiert nicht so „materialistisch“, daß er die Existenz des menschlichen Geistes leugnet, doch behauptet er, daß der Geist keine andere Existenzform besitze als die des Leibes. Diese anthropologische Grundentscheidung ist wichtig für die Medizinethik, da die Medizin es ihr zufolge in erster Linie mit dem menschlichen Leib und (was psychosomatische Überlegungen nicht aus- sondern einschließt) erst in zweiter Linie mit dem Geist zu tun hat. Sie ist aber auch wichtig für die Rechtstheorie, in der ein Dualismus von Geist und Leib als Dualismus von Kultur, Recht und Geschichte einerseits und der menschlichen Natur andererseits auftauchen könnte. Das Recht gehört Bonhoeffer zufolge nicht einer zweiten, höheren kulturellen Stufe "oberhalb“ des natürlichen Lebens an, sondern ist konkret auf die elementaren Lebensvoraussetzungen wie Leben, Sexualität, Essen, Trinken, Wohnen, Arbeit usw. bezogen. An ihnen sind die Rechte natürlichen Lebens orientiert; in ihnen hat positives Recht sich zu bewähren.

Wie vielfältig die mit der „Achtung der körperlichen Kontingenz des Menschen" betreffenden Fragen heute sind, kann der Blick in einen Grundgesetzkommentar zu Art.1 GG aufzeigen. Hier werden folgende Problemkomplexe aufgeführt: Folter, Mißhandlung und körperliche Strafen, Sexualität (z.B. körperliche Untersuchung einer Frau durch männliche Beamte), Ausforschung der Psyche mittels technischer Mittel (z.B. Lügendetektor), Menschenversuche, Schutz des menschlichen Körpers als Leichnam, Obduktion, pathologische Sektion, Organ-Transplantation, rechtliche Anerkennung transsexueller Prägung, Biotechnik, Reproduktionstechnik, Gentechnik, In-Vitro-Insemination, Leihmutterschaft, Selbstentfaltung der Mutter, verfassungsrechtliches Verbot anonymer Samenspenden, Ei-Diebstahl, Genomanalyse, Ermöglichung eines würdigen Todes $^{64}$.

Zwangssterilisation und „Euthanasie“ werden hier derart selbstverständlich als verfassungswidrig angesehen, daß man sie nicht einmal erwähnt. Gleichwohl zeigt die große Anzahl der Themen, daß die Frage nach den Rechten leiblichen Lebens durch gestiegene medizinische Manipulationsmöglichkeiten gegenüber den vierziger Jahren an Bedeutung nicht verloren, sondern gewonnen hat. Obwohl Dietrich Bonhoeffers ethische Theoriebildung auf Herausforderungen reagierte, die der Vergangenheit angehören, weist insbesondere seine These von der Würde des menschlichen Leibes über die zeitgeschichtlichen Zusammenhänge hinaus und ist darin aktuell geblieben.

${ }^{63}$ Vgl. Hartmann, Leib und Seele.

64 Vgl. Podlech, in: Azzola, Kommentar, zu Art. 1 Abs. 1, Rz. 44-54, S. 214-218. 
